\title{
COMT Polymorphisms and Anxiety-Related Personality Traits
}

\author{
Murray B Stein*,', Margaret Daniele Fallin ${ }^{2}$, Nicholas J Schork' and Joel Gelernter ${ }^{3}$ \\ 'Department of Psychiatry, University of California, San Diego, La Jolla, CA, USA; ${ }^{2}$ Department of Epidemiology, Johns Hopkins Bloomberg School \\ of Public Health, Baltimore, MD, USA; ${ }^{3}$ Department of Psychiatry, Yale University School of Medicine, New Haven, CT, USA
}

\begin{abstract}
High neuroticism and low extraversion are characteristic of anxiety-prone individuals. A functional variant in the catechol-Omethyltransferase (COMT) gene, the Vall58Met ('val/met') polymorphism, has been associated in some prior studies with several phenotypes, including neuroticism. We tested the hypothesis that the vall58met polymorphism would be associated with both high neuroticism and low extraversion, making it a plausible candidate locus for anxiety susceptibility. To determine whether vall 58 met is responsible for these effects, we also evaluated the association with haplotypes that included two other SNPs within the COMT gene. We collected a sample of 497 undergraduate college students who were phenotyped on a personality inventory (the NEO-Personality Inventory-Raised (NEO-PI-R)). Subjects were genotyped for three COMT polymorphisms: the well-studied nonsynonymous SNP rs4680 that generates a valine-to-methionine substitution (vall58met), rs737865 (near exon \#I), and rsI65599 (also functional, near the 3'UTR). Together, these three SNPs define a haplotype that is associated with reduced COMT expression in human brain. Logistic regression analyses were used to examine the effects of individual SNPs on extraversion and neuroticism scores. Score tests for association between these traits (quantitatively and dichotomously considered) and haplotypes were also conducted. We evaluated potential for population stratification artifact by genotyping a set of 36 unlinked highly polymorphic markers previously demonstrated to distinguish sufficiently ancestry of major American populations. Two of the SNPs (rs4680 ('val/met') and rs737865) were significantly associated with (low) extraversion and, less consistently, with (high) neuroticism, with effects confined to women. A significant association between COMT haplotype and (low) extraversion and (high) neuroticism was also observed. Formal testing showed that population structure did not explain the findings. These data suggest that involvement of the COMT locus in susceptibility to anxiety-related traits (ie low extraversion and high neuroticism) is unlikely to be wholly accounted for by the well-studied rs4680 ('val/met') polymorphism. Other functional variants may exist that contribute to this relationship. Possible sex-specific effects remain to be further studied and explained. Neuropsychopharmacology (2005) 30, 2092-2 102. doi:I 0. I038/sj.npp. I 300787; published online 8 June 2005
\end{abstract}

Keywords: anxiety; catechol-O-methyltransferase; polymorphism; genetics; personality

\section{INTRODUCTION}

Neuroticism is viewed as a risk factor (or personality template) for mood and anxiety disorders (Andrews et al, 2002; Hirschfeld et al, 1989; Kendler et al, 2002). Moreover, particularly in the case of major depression, there is evidence that neuroticism interacts with psychosocial stress to yield psychopathology (Kendler et al, 2003, 2004). As such, elucidating the genetics of personality traits such as neuroticism may contribute to a better understanding of the etiology of these disorders (Bienvenu and Stein, 2003; Fullerton et al, 2003; Reif and Lesch, 2003; Van Gestel and Van Broeckhoven, 2003).

*Correspondence: Dr MB Stein, Department of Psychiatry (0985), University of California, San Diego, 9500 Gilman Drive, La Jolla, CA 92093-0985, USA, Tel: + | 858622 6I24, Fax: + I 858450 |49|,

E-mail: mstein@ucsd.edu

Received 27 July 2004; revised 13 April 2005; accepted 26 April 2005 Online publication: 29 April 2005 at http://www.acnp.org/citations/ NPP04290504034I/default.pdf
Numerous studies have evaluated possible associations between candidate genes and neuroticism (and other anxiety-related traits). Most frequently studied in this regard has been the serotonin transporter promoter polymorphism (5-HTTLPR). Two recent meta-analyses (that included a substantially overlapping set of studies) found evidence of an association between 5-HTTLPR and neuroticism, although less so with other anxiety-related constructs (eg harm avoidance) (Schinka et al, 2004; Sen et al, 2004a). Other genes tested for an association with anxiety-related traits have included monoamine oxidase type A (Eley et al, 2003; Jorm et al, 2000), cytochrome P450 2D6 isoenzyme (Roberts et al, 2004), GABA-A receptor alpha-6 subunit (Sen et al, 2004b) and, in a number of studies, catechol-O-methyltransferase (COMT) (Eley et al, 2003; Enoch et al, 2003; Henderson et al, 2000).

Henderson and colleagues, in a community sample of 2237 Caucasians, found no association between a common COMT polymorphism (rs165688, also known as rs4680, and variously referred to in the literature as $\mathrm{val} / \mathrm{met}$ or val108met or val158met) and either neuroticism or anxiety 
or depressive symptoms (Henderson et al, 2000). In a sample of community subjects selected for either high or low on neuroticism, Eley et al (2003) found weak evidence for association with COMT val158met genotype, when females and males were considered separately. Another recent study of 101 patients with phobic disorders and 202 age-, sex-, and ethnicity-matched control subjects failed to find an association with val158met and phobic disorders (Samochowiec et al, 2004). In a sample of 1234 female nurses, McGrath and colleagues found an association of val158met genotype with phobic anxiety as measured by the Crown-Crisp experimental index, wherein homozygosity for the val allele was associated with a doubling of odds of having high phobic anxiety, compared to met homozygosity (McGrath et al, 2004).

When looking at patients with panic disorder, four of five association studies (one of which also provided evidence of linkage; Hamilton et al, 2002) have implicated COMT as a probable risk locus (Domschke et al, 2004; Hamilton et al, 2002; Ohara et al, 1998; Woo et al, 2002, 2004). Thus, the bulk of the published data implicate the COMT gene or a nearby region as a susceptibility locus for panic disorder and other anxiety-related traits, although the specific site(s) within the gene that confer this susceptibility, and the apparent sex-specific effects (in some studies), remain to be established.

The COMT gene (MIM 116790) has been widely speculated (and in some cases, shown) to be associated with a variety of neuropsychiatric disorders and putative endophenotypes beyond panic disorder. It has been extensively studied as a candidate gene for schizophrenia, with some replicated evidence of the involvement of the val/ met (rs4680) variant in the etiology of this syndrome (Glatt et al, 2003). (COMT also maps in the usual deletion region for velocardiofacial syndrome, which is itself associated with increased risk for schizophrenia; Pulver et al, 1994.) The COMT val allele is associated with less efficient prefrontal cognition in persons with schizophrenia (Goldberg et al, 2003), presumably through its established effects to increase prefrontal dopamine catabolism that are thought to contribute to an optimization or 'fine-tuning' of dopamine signaling, particularly under conditions of high dopamine load (Akil et al, 2003; Bilder et al, 2004; Seamans et al, 2001a,b; Winterer and Goldman, 2003). Although functional analysis of genetic variation in COMT suggests that rs4680 val activity is the predominant factor determining COMT activity in the prefrontal cortex (Chen et al, 2004a), other variants in the COMT gene have also been recently associated with schizophrenia. In particular, the rs165599 SNP (located near the $3^{\prime}$-UTR of the gene) has been strongly associated with schizophrenia, especially in women, in a large case-control study (Shifman et al, 2002). Those investigators also found that a haplotype consisting of rs165599-rs4680-rs737865 was very strongly associated with schizophrenia in their sample. In Irish families with a high density of schizophrenia, a different haplotype, composed of the same three markers but having only the 'val' allele in common, was recently reported to be preferentially transmitted to affected subjects (Chen et al, 2004b). These observations are of particular interest because this haplotype is associated with reduced COMT expression in human brain (Bray et al, 2003).
We therefore extended our investigation of the COMT gene and its relationship to neuroticism to include the three SNPs that comprise this haplotype (Figure 1). We also extended this area of inquiry by looking at another personality trait, extraversion. Although neuroticism has been studied much more extensively in relation to anxiety (and COMT), it could be argued that extraversion is of equal (or greater) relevance than neuroticism to certain anxiety disorders (eg agoraphobia and social phobia) (Bienvenu and Stein, 2003). Extraversion is defined as having high levels of sociability and preference for large groups and gatherings. Costa and McCrae (1992) describe individuals with high levels of extraversion as being assertive, active, talkative, upbeat, energetic, and optimistic. Persons with social phobia or agoraphobia tend to be very low in extraversion (Bienvenu et al, 2001), a trait that is at least as heritable as neuroticism (Jang et al, 1996, 1998). We have recently shown an influence of a variant in the beta-1adrenergic receptor gene on extraversion in a sample that overlaps substantially with the sample described herein (Stein et al, 2004).

Population subdivision is thought to be a major potential confound for case-control association studies (Freedman et al, 2004). We therefore used the method of Pritchard and Rosenberg (1999) to evaluate the potential for spurious association in our study sample that would result from population stratification (Figure 1).

\section{METHODS}

\section{Sample and Procedure}

Participants $(N=497)$ were recruited from among undergraduate psychology students at San Diego State University (SDSU) who had, in a group testing session (at which some questionnaires were completed and demographic information, including self-reported race/ethnicity, was provided), indicated their willingness to be contacted by research investigators to participate in future psychological experiments. They each came for a scheduled appointment at which a blood sample $(60 \mathrm{ml})$ was drawn for genetic studies and the subjects completed questionnaires and/or computerized tasks. Subjects gave informed, written consent to participate in this part of the study, which was approved by the Human Research Protection Programs at both SDSU and UCSD. Subjects received $\$ 25$ for providing the blood sample. Sociodemographic characteristics of the subjects are shown in Table 1 .

\section{Questionnaires}

NEO-Personality Inventory-Revised (NEO-PI-R) is a widely used, 240-item (plus three validity items) self-report

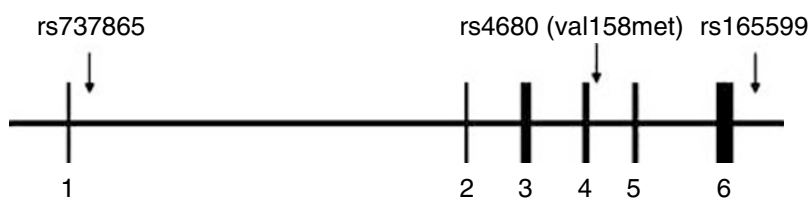

Figure I Relative locations of SNPs studied (not to scale). 
Table I Sociodemographic Characteristics of Study Subjects $(N=497)$

\begin{tabular}{lc}
\hline Variable & Distribution \\
\hline Age (years) (mean (SD)) & $18.9(2.2)$ \\
Sex (n) (male/female) & |54/343(31.0\%/69.0\%) \\
& \\
Race/ethnicity & \\
African American & $21(4.2 \%)$ \\
Asian American & $33(6.6 \%)$ \\
Hispanic American/Latino & $99(19.9 \%)$ \\
Native American & $2(0.4 \%)$ \\
Filipino American & $55(11.1 \%)$ \\
European American & $246(49.5 \%)$ \\
Other or missing & $41(8.2 \%)$ \\
\hline
\end{tabular}

measure of personality, grouped into five major domains: neuroticism $(\mathrm{N})$, extraversion $(\mathrm{E})$, openness to experience $(\mathrm{O})$, conscientiousness (C), and agreeableness (A) (Costa and McCrae, 1992). Each of the five domains also has six, lower-level facets. Some subjects completed a shorter (60item) version of the NEO, the NEO-Five Factor Inventory (NEO-FFI) (Costa and McCrae, 1992), which provides domain scores (expressed as $\mathrm{T}$ scores) that are highly correlated (Pearson's $r>0.90$ for each of the five domains) with those obtained from the full instrument, but for which more detailed facet-level scores cannot be obtained. T scores were calculated directly from college-age, sex-specific norms (Robert $\mathrm{R}$ McCrae $\mathrm{PhD}$, personal communication, November 14, 2001). As noted above, we limited our analyses to the two most widely studied NEO domains, E and $\mathrm{N}$, which also are likely to be most informative with regard to our understanding of genetic influences on anxiety-related traits (Bienvenu and Stein, 2003; Stein and Bienvenu, 2004).

\section{Genotype Analysis}

DNA was extracted from frozen whole blood using standard methods. Subjects were genotyped for three COMT polymorphisms: (1) the well-studied rs4680 polymorphism that produces a valine $\rightarrow$ methionine (val/met) substitution at codon 158 in the membrane-bound form of the COMT transcript; (2) rs737865, located in the first intron; and (3) rs165599, located near the $3^{\prime}$-UTR. Genotypes for rs4680 and rs165599 were obtained using PCR-RFLP techniques. Primers for rs4680 were $5^{\prime}$-agc cct ccg tgc tgc tgg agc tgg- $3^{\prime}$ and $5^{\prime}$-cat gcc ctc cct gcc cac agc cgg- $3^{\prime}$; the polymorphism was recognized with NlaIII digestion. Primers for rs165599 were $5^{\prime}$-gaa gga gat gct tcc act ctg $\mathrm{t}-3^{\prime}$ and $5^{\prime}$-aca ttc aaa gct ccc ctt gac- $3^{\prime}$; the polymorphism was recognized with $M s p$ I digestion. Restriction enzymes were purchased from New England Biolabs (Beverly, MA, USA). Gels were doublescored and double-entered. In all, $8 \%$ of genotypes were repeated at random as quality checks, with complete concordance. Genotypes for rs737865 were obtained using the ABI Taqman system as an 'assay-by-design' with primers (f) $5^{\prime}$-ggc ttg gag ggt cac ttt aaa caa $\mathrm{t}-3^{\prime}$ and (r) $5^{\prime}$-ccc tgc taa cag acc tgc ttt $\mathrm{t}-3^{\prime}$, and reporter sequences cac aaa aac ccc tgg ctg (vic) and aca caa aaa tcc ctg gct g (fam). Post-PCR fluorescence detection was accomplished with an ABI 7900 instrument. All Taqman genotypes were repeated in duplicate.

\section{Ancestry-Informative Marker Panel}

In all, 36 STR markers were used; selection and evaluation of these markers is described in detail elsewhere (Yang et al, 2005). First, we used the AmpFLSTR Identifiler PCR Amplification Kit (Applied Biosystems (ABI), Foster City, CA), marker details available at http://www.appliedbiosystems. $\mathrm{com} / \mathrm{catalog} / \mathrm{myab} /$ StoreCatalog/products/CategoryDetails.jsp? hierarchyID $=101 \&$ category 1 st $=111763 \&$ category 2 nd $=11177$ $8 \&$ category $3 \mathrm{rd}=111962$, which provides data from a set of 15 loci frequently used in forensic applications. Second, we selected 21 markers known to have high $\delta$ (calculated as the sum over all alleles of the absolute value of allele frequency difference between two populations divided by 2) between European Americans and African Americans, and in some cases Hispanic and Asian populations (Smith et al, 2001). Markers included were D1S196, D1S2628, D2S162, D2S319, D5S407, D5S410, D6S1610, D7S640, D7S657, D8S272, D8S1827, D9S175, D10S197, D10S1786, D11S935, D12S352, D14S68, D15S1002, D16S3017, D17S799, and D22S274. All STR markers were analyzed on an ABI PRISM 3100 semiautomated capillary fluorescence sequencer. Data were scored using Genemapper (ABI). The average missing data rate for ancestry-informative markers was $10 \%$.

\section{Data Analysis}

Most calculations, including linear regression analyses, were made using the biostatistical analysis program STATA (Version 8.2) (StataCorp, 2003). Between-group (ie, sex, ethnicity) differences in means are tested using Student's $t$-test; 95\% confidence intervals (CIS) for the differences between groups are also provided. Deviation from the genotype counts predicted by Hardy-Weinberg (HW) equilibrium expectations was tested with the installed user-written STATA package 'sg110' available at http:// www.stata.com/stb/stb48. The value of $D^{\prime}$, a standardized measure of linkage disequilibrium (LD) (Lewontin 1988), was computed. Allele frequencies were compared between the two largest ethnic groups in our sample (ie European American (EA) and Hispanic) using $\chi^{2}$ analysis.

Robust regression analyses were used to predict scores on the questionnaire measures using genotype as the independent variable. Power to detect a quantitative trait locus accounting for $2 \%$ of the phenotypic variance, given the sample size and frequency for the biallelic marker, was over 90\% (Purcell et al, 2003). Analysis of variance was also used to compare questionnaire scores by genotype, in order to demonstrate the existence of dose-response relationships. Odds ratios, on an allelic basis (Sasieni, 1997), were calculated to demonstrate the association between particular polymorphisms and extraversion scores (dichotomized into appropriate groupings); these tests were conducted with the installed user-written STATA package 'genassoc' 
written by David Clayton and available at http://wwwgene.cimr.cam.ac.uk/clayton/software/stata. Analyses stratified by sex are also reported, but stratification by selfreported race/ethnicity was not possible owing to the substantially reduced power for such analyses (eg sample size for Hispanics ranges from 86 to 99 for various analyses).

We used the haplo.score package (version 1.0.2, dated September 13, 2002; available at http://www.mayo.edu/ statgen). This software computes score statistics (which test the null hypothesis of no association of the trait with the genotype) to evaluate associations between ambiguous (reconstructed) haplotypes and traits (including quantitative traits) in unrelated subjects, and can consider the effects of nongenetic covariates (Schaid et al, 2002). The method is particularly useful when there may be more than one functional variant at the locus (as for COMT). An additional advantage is that speed of computation allows for empirical evaluation of statistical significance by means of simulation.

The Pritchard and Rosenberg stratification test statistic was computed with STRAT software (Pritchard et al, 2000). This test statistic (which is based on summing the $\chi^{2}$ test statistics for case-control comparisons at each of the unlinked stratification test loci) allows determination of whether cases and controls are appropriately matched; if mismatched, they are potentially subject to stratification error. Since this test can be performed only with binary (not quantitative) phenotypes, we recoded phenotypes to affection or nonaffection using a median split for these analyses. This should be a reasonable approximation, because the phenotypes (ie extraversion and neuroticism) followed a normal distribution (Figure 2).

\section{RESULTS}

\section{Behavioral Measures}

Distribution of NEO-E and NEO-N T-scores in the sample is shown in Figure 1. There were no significant differences in NEO-E scores between EA and Hispanic subjects, but EAs had significantly higher NEO-N scores (50.2 SD 11.9 vs 47.3 $\mathrm{SD} 10.5 ; \mathrm{t}=2.10, \mathrm{df}=343, p=0.04)$.

\section{COMT Allele and Genotype Frequencies}

There was no deviation from HW equilibrium expectations at any of the three loci for the overall sample. Distributions of the genotypes and alleles for all three loci are shown in Table 2. LD between the three loci was determined separately in EAs and Hispanics. In EAs: for rs 165599 and rs4680, Lewontin's $D^{\prime}=0.51$; for rs165599 and rs737865, $D^{\prime}=0.21 ;$ and for $\mathrm{rs} 4680$ and $\mathrm{r} 737865, D^{\prime}=0.73$. In Hispanics: for rs 165599 and rs4680, Lewontin's $D^{\prime}=0.96$; for rs165599 and rs737865, $D^{\prime}=0.05$; and for rs4680 and $\mathrm{r} 737865, D^{\prime}=0.58$.

Ethnic differences. There was no deviation from HW equilibrium expectations for any of the three polymorphic systems in either the EA or Hispanic subgroups. However, there were significant ethnic differences in the allelic distribution of all three systems: $\operatorname{rs} 16559\left(\chi^{2}=11.35\right.$, $\mathrm{df}=1, p<0.0005), \quad \mathrm{rs} 4680 \quad\left(\chi^{2}=7.09, \mathrm{df}=1, \quad p=0.008\right)$,

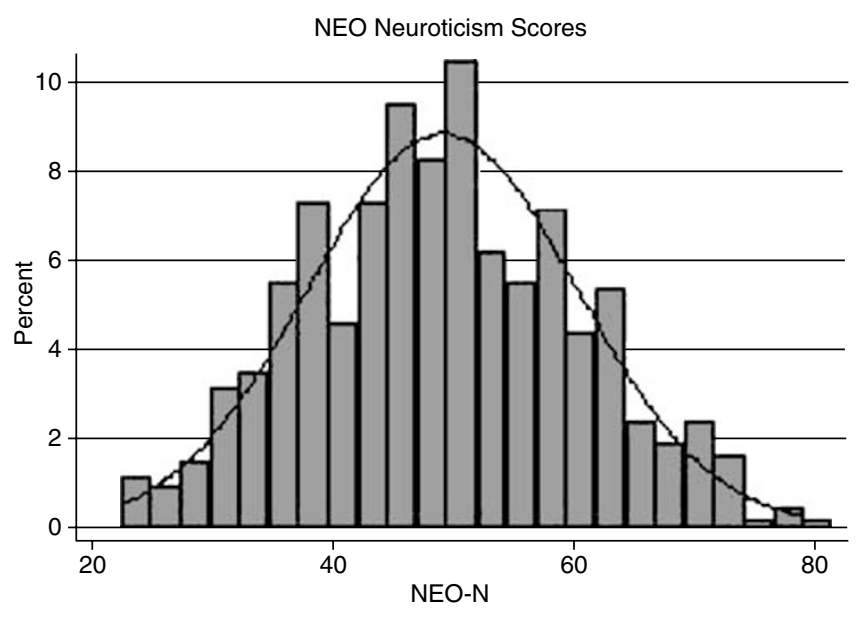

NEO Extraversion Scores

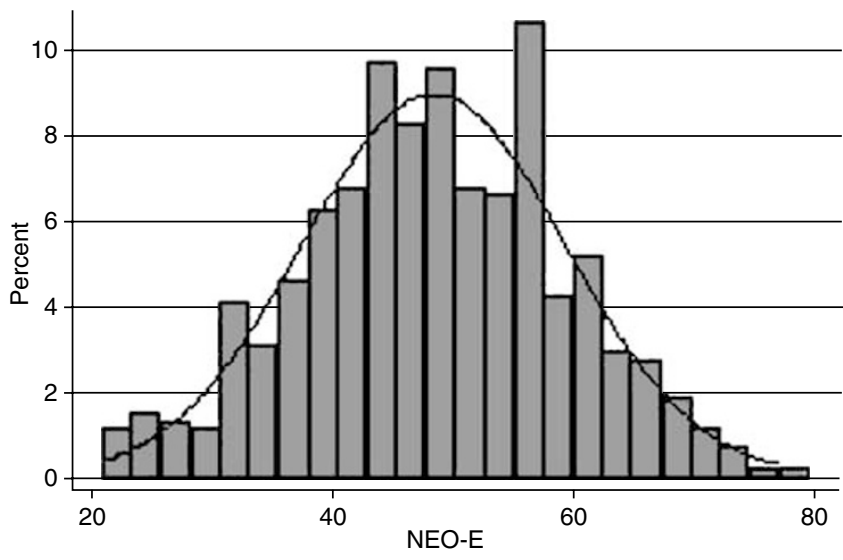

Figure 2 Distribution of NEO neuroticism (NEO-N; top) and extraversion (NEO-E; bottom) $T$-scores $(N=497)$.

and rs737865 $\left(\chi^{2}=8.77, \mathrm{df}=1, p=0.003\right)$ (Table 2). The EA genotype frequencies for rs165599 are similar to those reported elsewhere for 'Mixed Europeans' (Palmatier et al, 2004), for a large control (for breast cancer cases) sample of white Americans (Millikan et al, 1998), and for a large control (for schizophrenia cases) sample of Israelis of predominantly European descent (Shifman et al, 2002). The EA genotype frequencies for rs737865 in our study show a lower frequency of the rarer homozygous type (G/G) than in another study (Shifman et al, 2002); reasons for this difference are unclear. The EA rs4680 genotype frequencies in our study are also similar to those found in these two other studies (Millikan et al, 1998; Shifman et al, 2002).

\section{Population Stratification Test}

Extraversion. The two groups (ie created by split along median extraversion level for all subjects) were not significantly different by self-reported race/ethnicity $\left(\chi^{2}=2.79,5 \mathrm{df}, p=0.73\right) . N=441$ subjects with complete data; $\sum \chi^{2}=245.8$ ( $284 \mathrm{df}$ ), $p=$ NS. Thus, by this test, use of this entire sample with the extraversion phenotype should not be subject to stratification artifact.

Neuroticism. The two groups (ie created by split along median neuroticism level for all subjects) were not 
Table 2 Allele and Genotype Distribution of the COMT Receptor Polymorphisms

\begin{tabular}{|c|c|c|c|c|c|}
\hline \multirow[b]{2}{*}{ Variant } & \multicolumn{2}{|c|}{ Allele frequency (\%) } & \multicolumn{3}{|c|}{ Genotype frequency (\%) } \\
\hline & G & $\mathbf{A}$ & G/G & A/G & A/A \\
\hline Total sample $(N=473)$ & $252(0.266)$ & $694(0.734)$ & $28(0.059)$ & $196(0.4 \mid 4)$ & $249(0.526)$ \\
\hline European American only $(N=232)$ & $145(0.3 \mid 3)$ & $319(0.688)$ & $16(0.069)$ & I I 3 (0.487) & $103(0.444)$ \\
\hline Hispanic only $(N=93)$ & val (G) & met (A) & $\mathrm{val} / \mathrm{val}$ & $\mathrm{val} / \mathrm{met}$ & met/met \\
\hline \multicolumn{6}{|l|}{ rs4680 ('val/met') } \\
\hline Total sample $(N=497)$ & $591(0.595)$ & $403(0.405)$ & $185(0.372)$ & $221(0.445)$ & $91(0.183)$ \\
\hline European American only $(N=246)$ & $254(0.516)$ & $238(0.484)$ & $66(0.268)$ & $122(0.496)$ & $58(0.236)$ \\
\hline \multicolumn{6}{|l|}{ rs| 65599} \\
\hline Total sample $(N=46 \mid)$ & $372(0.404)$ & $550(0.597)$ & $81(0.176)$ & $210(0.456)$ & $170(0.369)$ \\
\hline European American only $(N=225)$ & I $52(0.338)$ & $298(0.662)$ & $25(0.111)$ & $102(0.453)$ & $98(0.436)$ \\
\hline Hispanic only $(N=86)$ & $84(0.488)$ & $88(0.5 \mid 2)$ & $22(0.256)$ & $40(0.465)$ & $24(0.279)$ \\
\hline
\end{tabular}

significantly different by self-reported race/ethnicity $\left(\chi^{2}=5.99,5 \mathrm{df}, p=0.31\right) . N=441$ subjects with complete data; $\Sigma \chi^{2} 265.6$ (284 df), $p=$ ns. Thus, by this test, use of this entire sample with the neuroticism phenotype should not be subject to stratification artifact.

\section{Association of Individual COMT Polymorphisms with Extraversion and Neuroticism}

rs4680 ( $\mathrm{val} / \mathrm{met}$ ).

Extraversion: For rs4680 ( $\mathrm{val} / \mathrm{met}$ ), presence of a met allele was associated with a higher odds of having low extraversion (ie being in the lower half of extraversion scores in this sample), although this failed to reach statistical significance $(\mathrm{OR}=1.24, \quad 95 \%$ CI $0.96-1.59$; $\left.\chi^{2}=2.68, \mathrm{df}=1, p=0.10\right)$. When considering genotype, being homozygous for the met allele was associated with a significantly higher odds of having low extraversion $\left(\mathrm{OR}=1.71,95 \%\right.$ CI $\left.1.05-2.82 ; \chi^{2}=5.23, \mathrm{df}=2, p=0.02\right)$. Mean NEO-E scores were 45.4 (SD 11.0) in met homozygotes compared to 48.3 (SD 11.4) in val hetero or homozygotes $(\mathrm{t}=2.28, \mathrm{df}=495, p=0.02)$.

When only women were considered, being homozygous for the met allele of rs4680 was associated with a significantly higher odds of having low extraversion $\left(\mathrm{OR}=2.66, \quad 95 \% \quad\right.$ CI $\quad 1.40-5.20 ; \quad \chi^{2}=10.42, \quad \mathrm{df}=1$, $p=0.001)$; when only men were considered, there was no significant association noted $\left(\chi^{2}=0.27, \mathrm{df}=1, p=0.6\right)$. Mean NEO-E scores were 43.2 (SD 10.6) in female met homozygotes compared to 47.9 (SD 11.8) in val hetero- or homozygotes $(\mathrm{t}=2.88, \mathrm{df}=341, p=0.004)$; there were no differences when only men were considered $(t=0.156$, $\mathrm{df}=152, p=0.9$ ).

Neuroticism: Being homozygous for the met allele was associated with a nonsignificantly higher odds of having high neuroticism, that is, being in the top half of the distribution of neuroticism in this sample $(\mathrm{OR}=0.70,95 \%$ $\mathrm{CI}=0.43-1.14 ; \chi^{2}=2.34, \mathrm{df}=1, p=0.13$ ), although this was statistically significant (and marginally so) in women only $\left(\mathrm{OR}=0.57,95 \%\right.$ CI $\left.0.31-1.05 ; \chi^{2}=3.79, \mathrm{df}=1, p=0.05\right)$. Mean NEO-N score in female met homozygotes was 52.4 (SD 11.5) compared to 48.2 (SD 11.5) in val hetero- or homozygotes $(\mathrm{t}=2.54, \mathrm{df}=341, p=0.01)$.

\section{rs737865.}

Extraversion: For rs737865, when considering men and women together, neither presence of an $A$ allele ( $\mathrm{OR}=1.23$, $95 \%$ CI $0.92-1.63 ; \chi^{2}=1.99$, df $=1, p=0.16$ ) nor, when considering genotype, being homozygous for the $A$ allele $\left(\mathrm{OR}=1.26,95 \%\right.$ CI $\left.0.87-1.84 ; \chi^{2}=1.60, \mathrm{df}=2, p=0.21\right)$, was associated with a significantly higher odds of having low extraversion.

However, when only women were considered, being homozygous for the $A$ allele of rs737865 was associated with a marginally significantly higher odds of having low extraversion $\left(\mathrm{OR}=1.53,95 \%\right.$ CI $0.97-2.42 ; \chi^{2}=3.77, \mathrm{df}=1$, $p=0.052$ ). Mean NEO-E scores were 45.5 (SD 12.2) in $A$ homozygotes compared to 48.7 (SD 10.8) in $G$ hetero- or homozygotes $(t=2.62, \mathrm{df}=332, p=0.0093)$; there were no significant differences when only men were considered $(\mathrm{t}=0.83, \mathrm{df}=140, p=0.41)$. 
Neuroticism: There were no significant associations between rs737865 and neuroticism at either the allelic or genotypic level.

rs165599. For rs165599, there were no significant associations with extraversion or neuroticism at either the allelic or genotypic level.

\section{Haplotype Analyses}

Extraversion. Score tests using haplo.score (Schaid et al, 2002) revealed a consistent association between extraversion as a dichotomous trait (split along the median for this sample, as described above) or as a quantitative trait and one or more of the haplotypes (dichotomous trait global score statistic $=16.38, \mathrm{df}=7, p=0.02$; quantitative trait global score statistic $=13.27, \mathrm{df}=7, \quad p=0.07$; a rare haplotype with estimated frequency $<0.005$ was excluded from the analyses). Haplotype-specific scores are as shown in Tables $3 \mathrm{a}$ and $3 \mathrm{~b}$. In both the dichotomous (Table 3a) and quantitative trait (Table $3 \mathrm{~b}$ ) analyses, two of the haplotypes (rs737865 ' $A$ '-rs4680 ' $v a l$ '-rs16599' $A$ ' and rs737865 ' $G$ '-rs4680 ' $m e t^{\prime}$-rs16599 ' $A$ ') were associated with higher NEO-E scores, whereas a third haplotype (rs737865 ' $A$ '-rs4680 ' $m e t^{\prime}-\mathrm{rs} 16599$ 'A') was associated with lower NEO-E scores. When rerun separately for women and men, the same three haplotypes emerged as being significantly associated with extraversion in women only (absolute value of haplotype scores ranged from 2.06 to 2.45 , all $p$-values $<0.05$ ) (Tables $3 \mathrm{a}$ and $3 \mathrm{~b}$ ).

Neuroticism. Score tests revealed an association between neuroticism as a dichotomous trait (split along the median for this sample, as described above) (Table 4a) or as a quantitative trait (in women only) (Table $4 \mathrm{~b}$ ) and one or more of the haplotypes (dichotomous trait global score statistic $=13.55, \mathrm{df}=7, p=0.056$ and simulated $p=0.03$; quantitative trait global score statistic in women only $=11.89, \mathrm{df}=7, p=0.10$; a rare haplotype with frequency $<0.005$ was excluded from the analyses). In the dichotomous trait analysis (in the entire sample and in women only), a different haplotype (rs737865 ' $G$ '-rs4680 ' $m e t$ '-rs16599' $A$ ') was associated with higher neuroticism (haplotype score $=2.87$ in the entire sample and 2.82 in women only, both $p<0.005)$ (Table $4 \mathrm{a})$. In the quantitative trait analyses in women only, the same three 3-SNP haplotypes that showed an association with extraversion were implicated (haplotype scores ranging from 1.6 to 2.0, with $p$-values ranging from 0.04 to 0.10 ) (Table $4 \mathrm{~b}$ ).

\section{DISCUSSION}

Studying normative personality has relevance for the understanding of the genetic basis of disordered personality (Jang and Livesley, 1999). In this study, we found that polymorphisms of the COMT gene were associated with variation in two traits that are over- and underexpressed, respectively, in anxiety (and some mood) disorders: neuroticism and extraversion (Bienvenu and Stein, 2003; Stein and Bienvenu, 2004). One of the polymorphisms, rs4680 (often referred to in the literature as 'va108met'), thought to be functional on the basis of its effects on catecholamine metabolism in animal studies (Matsumoto et al, 2003) and its dose-response relationship to executive function in humans (Goldberg et al, 2003), has been the most widely studied. In many studies over the past halfdecade, variation in val158met has been associated with a variety of personality traits such as harm avoidance (Enoch

Table 3a Haplotype-Specific Scores for Extraversion as a Dichotomous Trait (Median Split)

\begin{tabular}{|c|c|c|c|c|c|c|c|}
\hline & rs737865 & vall58met & rs165599 & Frequency & Score & $p$ & Sim. $p$ \\
\hline \multicolumn{8}{|c|}{ I. Entire sample $(N=452)^{a}$} \\
\hline$[1]$ & A & $A$ & $A$ & 0.315 & 1.80 & $0.07 \mid$ & 0.07 \\
\hline [2] & $A$ & G & G & 0.239 & 0.61 & 0.54 & 0.54 \\
\hline [3] & $A$ & $A$ & G & 0.058 & 1.10 & 0.270 & 0.25 \\
\hline [4] & G & G & G & 0.106 & -0.21 & 0.83 & 0.80 \\
\hline$[7]$ & A & G & A & 0.123 & -2.32 & 0.02 & 0.02 \\
\hline \multicolumn{8}{|c|}{ II. Women only $(N=347)^{b}$} \\
\hline$[1]$ & A & A & A & 0.305 & 2.06 & 0.04 & 0.04 \\
\hline [6] & G & A & A & 0.040 & -2.45 & 0.01 & 0.02 \\
\hline [7] & A & G & $A$ & 0.108 & -2.26 & 0.02 & 0.03 \\
\hline
\end{tabular}

${ }^{\mathrm{a}}$ Global statistic $=16.4, \mathrm{df}=7, p=0.02$ (simulated $\left.p=0.02\right)$.

${ }^{\mathrm{b}}$ Global statistic $=20.5, \mathrm{df}=7, p=0.005$ (simulated $p=0.002$ ). 
Table 3b Haplotype-Specific Scores for Extraversion as a Quantitative Trait

\begin{tabular}{|c|c|c|c|c|c|c|c|}
\hline & rs737865 & vall58met & rsI65599 & Frequency & Score & $p$ & Sim. $p$ \\
\hline \multicolumn{8}{|c|}{ 1. Entire sample $(N=452)^{\mathrm{a}}$} \\
\hline$[1]$ & A & A & $A$ & 0.315 & -2.47 & 0.02 & 0.02 \\
\hline [2] & $A$ & G & G & 0.239 & -0.548 & 0.58 & 0.54 \\
\hline [3] & A & A & G & 0.058 & -0.451 & 0.65 & 0.65 \\
\hline$[4]$ & G & G & G & 0.106 & 0.786 & 0.43 & 0.42 \\
\hline [6] & G & $A$ & $A$ & 0.027 & 1.73 & 0.08 & 0.07 \\
\hline [7] & A & G & A & 0.123 & 1.76 & 0.08 & 0.08 \\
\hline \multicolumn{8}{|c|}{ II. Women only $(N=347)^{b}$} \\
\hline$[1]$ & A & A & A & 0.305 & -2.44 & 0.02 & 0.01 \\
\hline [6] & G & $A$ & A & 0.040 & 1.94 & 0.05 & 0.04 \\
\hline$[7]$ & A & G & $A$ & 0.108 & 1.61 & 0.11 & 0.13 \\
\hline
\end{tabular}

${ }^{a}$ Global statistic $=13.3, \mathrm{df}=7, p=0.07$ (simulated $p=0.07$ ).

${ }^{\mathrm{b}}$ Global statistic $=16.5, \mathrm{df}=7, p=0.02$ (simulated $p=0.04$ ).

Table 4a Haplotype-Specific Scores for Neuroticism as a Dichotomous Trait (Median Split)

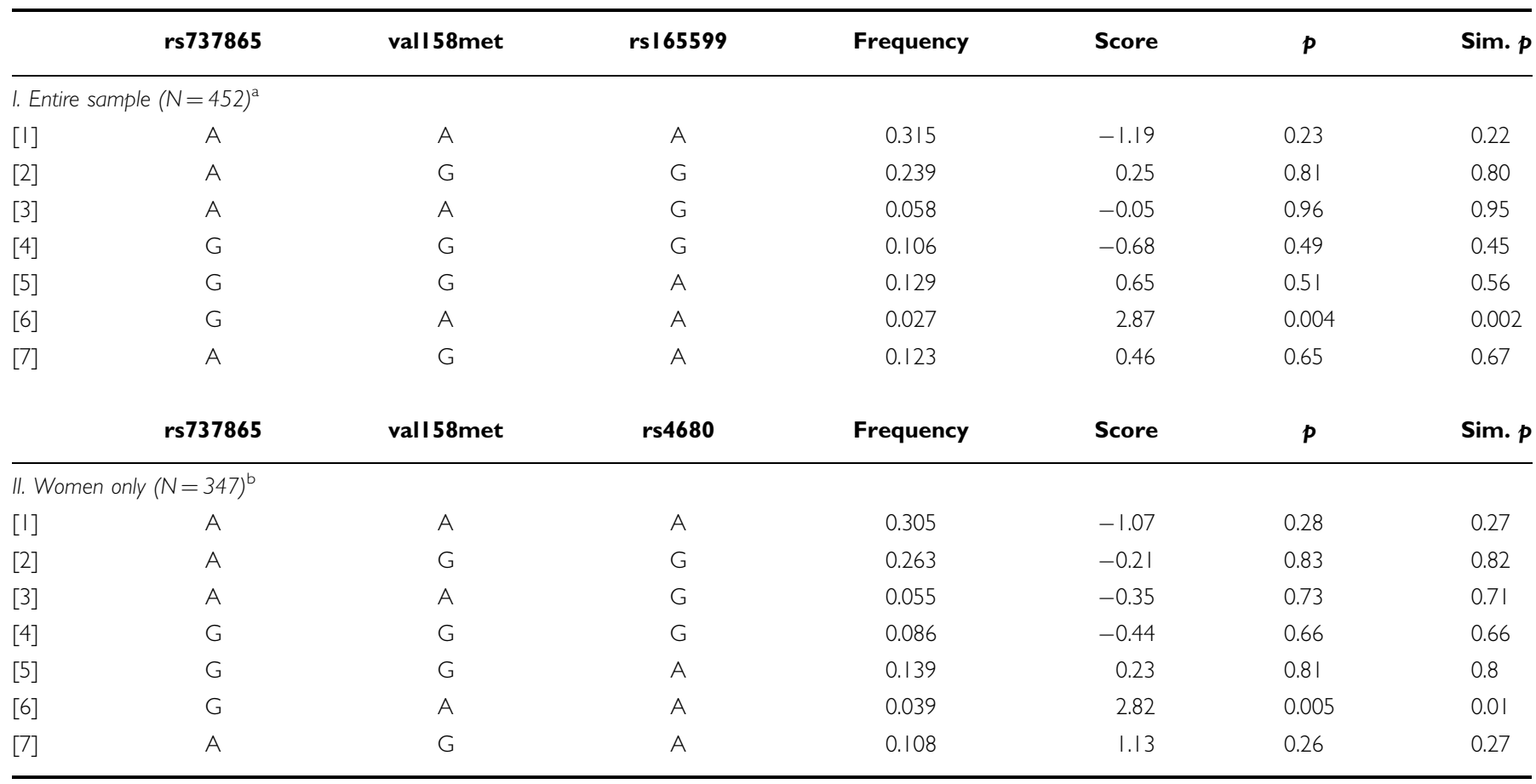

${ }^{\mathrm{a}}$ Global statistic $=13.5, \mathrm{df}=7, p=0.06$ (simulated $p=0.03$ ).

${ }^{\mathrm{b}}$ Global statistic $=13.2, \mathrm{df}=7, p=0.07$ (simulated $p=0.06$ ).

et al, 2003), neuroticism (Eley et al, 2003) (but not in all studies; Henderson et al, 2000), and aggression (Rujescu et al, 2003). It has also been strongly associated with one anxiety disorder in particular - panic disorder (Domschke et al, 2004; Hamilton et al, 2002; Woo et al, 2002,
2004) - but not with a mixture of anxiety-phobic disorders in several other studies (Ohara et al, 1998; Samochowiec et al, 2004) and, according to a recent meta-analysis, not with obsessive-compulsive disorder (Azzam and Mathews, 2003). 
Table 4b Haplotype-Specific Scores for Neuroticism as a Quantitative Trait

\begin{tabular}{|c|c|c|c|c|c|c|c|}
\hline & rs737865 & vall 58met & rs4680 & Frequency & Score & $p$ & Sim. $p$ \\
\hline \multicolumn{8}{|c|}{ I. Entire sample $(N=452)^{\mathrm{a}}$} \\
\hline$[1]$ & A & A & A & 0.315 & 1.34 & 0.18 & 0.158 \\
\hline$[2]$ & $A$ & G & G & 0.239 & -0.94 & 0.35 & 0.308 \\
\hline$[3]$ & A & A & G & 0.058 & -0.39 & 0.70 & 0.678 \\
\hline$[4]$ & G & G & G & 0.106 & 0.68 & 0.50 & 0.492 \\
\hline [6] & G & A & A & 0.027 & -1.59 & 0.11 & 0.13 \\
\hline [7] & A & G & A & 0.123 & -1.23 & 0.22 & 0.224 \\
\hline \multicolumn{8}{|c|}{ II. Women only $(N=347)^{b}$} \\
\hline$[1]$ & A & A & A & 0.305 & 1.96 & 0.05 & 0.04 \\
\hline [6] & G & A & A & 0.039 & -1.63 & 0.10 & 0.10 \\
\hline [7] & A & G & A & 0.108 & -1.76 & 0.08 & 0.06 \\
\hline
\end{tabular}

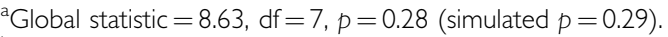

${ }^{b}$ Global statistic $=11.9, \mathrm{df}=7, p=0.10($ simulated $p=0.12)$.

There was therefore ample a priori reason to hypothesize that we would find an effect of the val158met polymorphism on neuroticism and extraversion in this study. And although val158met is by far the most widely studied of the COMT polymorphisms, evidence is emerging that other loci either within or near the COMT gene may confer susceptibility to panic disorder (Hamilton et al, 2002) and schizophrenia (Palmatier et al, 2004; Shifman et al, 2002). In the case of schizophrenia, a particular functional (Bray et al, 2003) 3-SNP haplotype consisting of the three loci studied here was strongly associated with schizophrenia (Shifman et al, 2002). We therefore considered the possibility that this haplotype might be associated with extraversion and neuroticism in our sample. This provided us with the opportunity to test the hypothesis that loci other than (or in addition to) the val158met locus might be associated with these traits.

In analyses on a SNP-by-SNP basis, we found that two of the three loci studied-val158met and rs737865-were associated with variation in extraversion and, somewhat less consistently, neuroticism. When the 3-SNP haplotypes were considered, we found evidence of modest influence of several of the haplotypes on extraversion levels (and to a lesser extent, on neuroticism levels). The haplotype analyses showed that neither variation in val158met nor 737865 alone could explain the relationship with extraversion: SNPs that contained alternate versions of either SNP were noted to influence the trait in ways that would be inconsistent with single-locus effects (eg haplotypes that influenced extraversion in a particular direction could contain either the val or the met variant of val158met). Thus, these observations using haplotypes (Fallin et al, 2001; Martin et al, 2000) strongly suggest that the most powerful (if not the sole) influences on extraversion (and, to a lesser extent, neuroticism) are contributed by loci that either lie between rs737865 and val158met, or lie elsewhere within the COMT gene or nearby genes in linkage disequilibrium with these loci. Similar conclusions have recently been reached in the case of schizophrenia, where it has been recognized that val/ met is unlikely to be the disease causing gene (Handoko et al, 2004). Resequencing efforts may provide evidence to support this hypothesis.

Of particular interest is our observation that the genetic associations to COMT (when observed from either a singleor multi haplotype perspective) were confined to women. An effect of val158met on harm avoidance in women was also reported to be confined to women (Enoch et al, 2003) and, to the extent that the combination of low extraversion and high neuroticism correspond to high harm avoidance, our observations can be considered to be a replication of those results. Furthermore, several studies have found associations of val158met and panic disorder specific to women (Domschke et al, 2004; Woo et al, 2004). These results also fit well with the fact that most anxiety (and depressive) disorders are more common in women, and are consistent with preclinical data indicating the possibility for sexually dimorphic effects of COMT variants on catecholamine metabolism (Huotari et al, 2002). This observation must be interpreted with caution, however, because there were more than twice as many women as men in our sample, so we might have lacked power to detect a signal in men. Replication of our results in larger samples will be required.

Our study has a number of additional limitations and caveats. We chose three markers based on prior knowledge that they generated the most meaningful haplotype information in a previous study (Shifman et al, 2002). However, we did not genotype all known COMT SNPs, and 
we therefore could have missed true associations with other variants. Although the number included is sufficient to enable us to fairly confidently exclude val158met as a sufficient explanation for the findings, it is insufficient to enable us to further localize influential loci to other DNA sequence variations in COMT or even to other nearby genes that could be in LD with the SNPs (and haplotypes) observed here. This will require more extensive genotyping of multiple COMT polymorphic sites. Other limitations are shared by many other studies that aim to associate complex phenotypes with specific genetic variants: relatively small sample size, uncertain prior odds of association (Sullivan et al, 2001), and testing of multiple phenotypes against multiple genetic polymorphisms (Colhoun et al, 2003; Hirschhorn et al, 2002; Ioannidis et al, 2003). Given that our study focused on a single gene and its relationship to these two well-studied personality traits (ie we were not intending to make multiple inferences), some biostatisticians would argue that in this context no adjustments are needed for multiple comparisons (Rothman, 1990; Saritz and Olshan, 1995). However, each of these limitations is sufficiently important that our findings must be considered preliminary until they are more widely replicated.

Also, we studied a racially mixed sample, and in the course of the work demonstrated significant differences in allele distribution for all three variants studied by selfreported race. Even prior to the formal test, we felt that we were at low risk for stratification artifact because extraversion, the phenotype with the strongest and most consistent findings in this study, was not observed to be significantly different among Hispanics and EAs (the two groups large enough to permit comparison), although such differences did appear for neuroticism. However, without a formal test for stratification, it could also have been argued that in the worst case, this could have led to a false positive finding via population stratification (Freedman et al, 2004). However, using a set of 36 markers including 21 that were selected specifically for their population-distinguishing characteristics, we tested formally for possible effects of population structure in creating spurious association, and were able to exclude this possibility. Still, even though we have shown previously that the 36 marker set separates out clusters that correspond well to self-reported race/ethnicity in this sample (Stein et al, 2004) and in other samples (Yang et al, 2005), we cannot exclude the possibility that more subtle heterogeneity exists than can be accounted for with this limited number of markers (Burchard et al, 2003). On the other hand, given the extremely high correspondence between self-identified race/ethnicity and genetic clusters and the lack of evidence of substantial subgroups within these clusters (Tang et al, 2005), the potential for confounding in these populations - while not eliminatedis certainly mitigated. Furthermore, the fact that our Hispanic subjects - the ethnic group with the greatest within-group genetic differentiation on the basis of current geographic location-were all sampled from the same geographic region (Southern California) also provides some degree of reassurance in this regard (Tang et al, 2005).

Our finding that common variants in COMT are associated with variation in common personality traits is not inconsistent with the notion that common genetic variants are likely to influence common diseases (or traits)
(Lohmueller et al, 2003). The magnitude of effects attributable to the COMT variants studied here was, however, quite small, raising the question of what role, if any, these variants might play as susceptibility factors for psychopathology. Thus, in addition to seeing our work replicated by other research groups in other samples, a next step to confirm the relevance of COMT variants to mental disorders will be to test its importance, through association and linkage studies, to other disorders characterized by extremely low extraversion, for example, generalized social phobia (Jang et al, 2001; Stein et al, 2001).

\section{ACKNOWLEDGEMENTS}

We are grateful to Shadha Hami, MA for coordinating the study and providing database management expertise; and to Ann Marie Lacobelle and Greg Kay for excellent technical assistance. This work was supported in part by NIMH Grant MH64122 to MBS and NIAAA Grants AA11330, and NIDA Grants DA12690, DA12849, and DA15105 to JG; and the US Department of Veterans Affairs (the VA Medical Research Program (Merit Review) to JG).

\section{CONFLICT OF INTEREST}

None of the authors declare any financial or other form of conflict of interest with the content of this manuscript.

\section{REFERENCES}

Akil M, Kolachana BS, Rothmond DA, Hyde TM, Weinberger DR, Kleinman JE (2003). Catechol-O-methyltransferase genotype and dopamine regulation in the human brain. J Neurosci 23: 2008-2013.

Andrews G, Slade T, Issakidis C (2002). Deconstructing current comorbidity: data from the Australian National Survey of Mental Health and Well-Being. Br J Psychiatry 181: 306-314.

Azzam A, Mathews CA (2003). Meta-analysis of the association between the catecholamine-O-methyl-transferase gene and obsessive-compulsive disorder. Am J Med Genet 123B: 64-69.

Bienvenu OJ, Nestadt G, Samuels JF, Howard WT, Costa Jr PT, Eaton WW (2001). Phobic, panic, and major depressive disorders and the five-factor model of personality. J Nerv Ment Dis 189: 154-161.

Bienvenu OJ, Stein MB (2003). Personality and anxiety disorders: a review. J Pers Disord 17: 139-151.

Bilder RM, Volavka J, Lachman HM, Grace AA (2004). The catechol-O-methyltransferase polymorphism: relations to the tonic-phasic dopamine hypothesis and neuropsychiatric phenotypes. Neuropsychopharmacology 29: 1943-1961.

Bray NJ, Buckland PR, Williams NM, Williams HJ, Norton N, Owen MJ et al (2003). A haplotype implicated in schizophrenia susceptibility is associated with reduced COMT expression in human brain. Am J Hum Genet 73: 152-161.

Burchard EG, Ziv E, Coyle N, Gomez SL, Tang H, Karter AJ et al (2003). The importance of race and ethnic background in biomedical research and clinical practice. $N$ Engl J Med 348: $1170-1175$.

Chen J, Lipska BK, Halim N, Ma QD, Matsumoto M, Melhem S et al (2004a). Functional analysis of genetic variation in catechol- $O$ methyltransferase (COMT): effects on mRNA, protein, and enzyme activity in postmortem human brain. Am J Hum Genet 75: 807-821. 
Chen X, Wang X, O’Neill AF, Walsh D, Kendler KS (2004b). Variants in the catechol-o-methyltransferase (COMT) gene are associated with schizophrenia in Irish high-density families. Mol Psychiatry 9: 962-967.

Colhoun HM, McKeigue PM, Smith GD (2003). Problems of reporting genetic associations with complex outcomes. Lancet 361: 865-872.

Costa Jr PT, McCrae RR (1992). NEO PI-R Professional Manual. Psychological Assessment Resources, Inc.: Odessa, FL.

Domschke K, Freitag CM, Kuhlenbumer G, Schirmacher A, Sand P, Nyhuis $\mathrm{P}$ et al (2004). Association of the functional V158M catechol-O-methyl-transferase polymorphism with panic disorder in women. Int J Neuropsychopharmacol 7: 1-6.

Eley TC, Tahir E, Angleitner A, Harriss K, McClay J, Plomin R et al (2003). Association analysis of MAOA and COMT with neuroticism assessed by peers. Am J Med Genet 120B: 90-96.

Enoch MA, Xu K, Ferro E, Harris CR, Goldman D (2003). Genetic origins of anxiety in women: a role for a functional catechol-Omethyltransferase polymorphism. Psychiatr Genet 13: 33-41.

Fallin D, Cohen A, Essioux L, Chumakov I, Blumenfeld M, Cohen $\mathrm{D}$ et al (2001). Genetic analysis of case/control data using estimated haplotype frequencies: application to APOE locus variation and Alzheimer's disease. Genome Res 11: 143-151.

Freedman ML, Reich D, Penney KL, McDonald GJ, Mignault AA, Patterson $\mathrm{N}$ et al (2004). Assessing the impact of population stratification on genetic association studies. Nat Genet 36: 388-393.

Fullerton J, Cubin M, Tiwari H, Wang C, Bomhra A, Davidson S et al (2003). Linkage analysis of extremely discordant and concordant sibling pairs identifies quantitative-trait loci that influence variation in the human personality trait neuroticism. Am J Hum Genet 72: 879-890.

Glatt SJ, Faraone SV, Tsuang MT (2003). Association between a functional catechol $o$-methyltransferase gene polymorphism and schizophrenia: meta-analysis of case-control and family-based studies. Am J Psychiatry 160: 469-476.

Goldberg TE, Egan MF, Gscheidle T, Coppola R, Weickert T, Kolachana BS et al (2003). Executive subprocesses in working memory: relationship to catechol-O-methyltransferase Val158Met genotype and schizophrenia. Arch Gen Psychiatry 60: 889-896.

Hamilton SP, Slager SL, Heiman GA, Deng Z, Haghighi F, Klein DF et al (2002). Evidence for a susceptibility locus for panic disorder near the catechol-O-methyltransferase gene on chromosome 22 . Biol Psychiatry 51: 591-601.

Handoko HY, Nyholt DR, Hayward NK, Nertney DA, Hannah DE, Windus LC et al (2004). Separate and interacting effects within the catechol-O-methyltransferase (COMT) are associated with schizophrenia. Mol Psychiatry [Epub ahead of print].

Henderson AS, Korten AE, Jorm AF, Jacomb PA, Christensen H, Rodgers B et al (2000). COMT and DRD3 polymorphisms, environmental exposures, and personality traits related to common mental disorders. Am J Med Genet 96: 102-107.

Hirschfeld RM, Klerman GL, Lavori P, Keller MB, Griffith P, Coryell W (1989). Premorbid personality assesssments of first onset of major depression. Arch Gen Psychiatry 46: 345-350.

Hirschhorn JN, Lohmueller K, Byrne E, Hirschhorn K (2002). A comprehensive review of genetic association studies. Genet Med 4: 45-61.

Huotari M, Gogos JA, Karayiorgou M, Koponen O, Forsberg M, Raasmaja A et al (2002). Brain catecholamine metabolism in catechol-O-methyltransferase (COMT)-deficient mice. Eur J Neurosci 15: 246-256.

Ioannidis JP, Trikalinos TA, Ntzani EE, Contopoulos-Ioannidis DG (2003). Genetic associations in large versus small studies: an empirical assessment. Lancet 361: 567-571.

Jang KL, Livesley WJ (1999). Why do measures of normal and disordered personality correlate? A study of genetic comorbidity. J Pers Disord 13: 10-17.
Jang KL, Livesley WJ, Vernon PA (1996). Heritability of the big five personality dimensions and their facets: a twin study. J Pers 64: 577-591.

Jang KL, McCrae RR, Angleitner A, Riemann R, Livesley WJ (1998). Heritability of facet-level traits in a cross-cultural twin sample: support for a hierarchical model of personality. J Pers Soc Psychol 74: 1556-1565.

Jang KL, Vernon PA, Livesley WJ, Stein MB, Wolf H (2001). Intraand extra-familial influences on alcohol and drug misuse: a twin study of gene-environment correlation. Addiction 96: 1307-1318.

Jorm AF, Henderson AS, Jacomb PA, Christensen H, Korten AE, Rodgers B et al (2000). Association of a functional polymorphism of the monoamine oxidase A gene promoter with personality and psychiatric symptoms. Psychiatr Genet 10: 87-90. Kendler KS, Gardner CO, Prescott CA (2003). Personality and the experience of environmental adversity. Psychol Med 33: 1193-1202.

Kendler KS, Kuhn J, Prescott CA (2004). The interrelationship of neuroticism, sex, and stressful life events in the prediction of episodes of major depression. Am J Psychiatry 161: 631-636.

Kendler KS, Myers J, Prescott CA (2002). The etiology of phobias: an evaluation of the stress-diathesis model. Arch Gen Psychiatry 59: $242-248$.

Lewontin R (1988). On measures of gametic disequilibrium. Genetics 120: 849-852.

Lohmueller KE, Pearce CL, Pike M, Lander ES, Hirschhorn JN (2003). Meta-analysis of genetic association studies supports a contribution of common variants to susceptibility to common disease. Nat Genet 33: 177-182.

Martin ER, Lai EH, Gilbert JR, Rogala AR, Afashari AJ, Riley J et al (2000). SNPing away at complex disease: analysis of singlenucleotide polymorphisms around APOE in Alzheimer disease. Am J Hum Genet 67: 383-394.

Matsumoto M, Weickert CS, Akil M, Lipska BK, Hyde TM, Herman $\mathrm{MM}$ et al (2003). Catechol O-methyltransferase mRNA expression in human and rat brain: evidence for a role in cortical neuronal function. Neuroscience 116: 127-137.

McGrath M, Kawachi I, Ascherio A, Colditz GA, Hunter DJ, De Vivo I (2004). Association between catechol-O-methyltransferase and phobic anxiety. Am J Psychiatry 161: 1703-1705.

Millikan RC, Pittman GS, Tse CK, Duell E, Newman B, Savitz D et al (1998). Catechol-O-methyltransferase and breast cancer risk. Carcinogenesis 19: 1943-1947.

Ohara K, Nagai M, Suzuki Y, Ochiai M, Ohara K (1998). No association between anxiety disorders and catechol-O-methylransferase polymorphism. Psychiatry Res 80: 145-148.

Palmatier MA, Pakstis AJ, Speed W, Paschou P, Goldman D, Odunsi A et al (2004). COMT haplotypes suggest P2 promoter region relevance for schizophrenia. Mol Psychiatry 9: 859-870.

Pritchard JK, Rosenberg NA (1999). Use of unlinked genetic markers to detect population stratification in association studies. Am J Hum Genet 65: 220-228.

Pritchard JK, Stephens M, Donnely P (2000). Inference of population structure using multilocus genotype data. Genetics 155: 945-959.

Pulver AE, Nestadt G, Goldberg R, Shprintzen RJ, Lamacz M, Wolyniec PS et al (1994). Psychotic illness in patients diagnosed with velo-cardio-facial syndrome and their relatives. J Nerv Ment Dis 182: 476-478.

Purcell S, Cherny SS, Sham PC (2003). Genetic Power Calculator: design of linkage and association genetic mapping studies of complex traits. Bioinformatics 19: 149-150.

Reif A, Lesch KP (2003). Toward a molecular architecture of personality. Behav Brain Res 139: 1-20.

Roberts RL, Luty SE, Mulder RT, Joyce PR, Kennedy MA (2004). Association between cytochrome P450 2D6 genotype and harm avoidance. Am J Med Genet 127B: 90-93. 
Rothman KJ (1990). No adjustments are needed for multiple comparisons. Epidemiology 1: 43-46.

Rujescu D, Giegling I, Gietl A, Hartmann AM, Moller HJ (2003). A functional single nucleotide polymorphism (V158M) in the COMT gene is associated with aggressive personality traits. Biol Psychiatry 54: 34-39.

Samochowiec J, Hajduk A, Samochowiec A, Horodnicki J, Stepien G, Grzywacz A et al (2004). Association studies of MAO-A, COMT, and 5-HTT genes polymorphisms in patients with anxiety disorders of the phobic spectrum. Psychiatry Res 128: 21-26.

Saritz DA, Olshan AF (1995). Multiple comparisons and related issues in epidemiologic research. Am J Epidemiol 142: 904-908.

Sasieni PD (1997). From genotypes to genes: doubling the sample size. Biometrics 53: 1253-1261.

Schaid DJ, Rowland CM, Tines DE, Jacobson RM, Poland GA (2002). Score tests for association between traits and haplotypes when linkage phase is ambiguous. Am J Hum Genet 70: 425-434.

Schinka JA, Busch RM, Robichaux-Keene N (2004). A metaanalysis of the association between the serotonin transporter gene polymorphism (5-HTTLPR) and trait anxiety. Mol Psychiatry 9: 197-202.

Seamans JK, Durstewitz D, Christie BR, Stevens CF, Sejnowski TJ (2001a). Dopamine D1/D5 receptor modulation of excitatory synaptic inputs to layer V prefrontal cortex neurons. Proc Natl Acad Sci USA 98: 301-306.

Seamans JK, Gorelova N, Durstewitz D, Yang CR (2001b). Bidirectional dopamine modulation of GABAergic inhibition in prefrontal cortical pyramidal neurons. J Neurosci 21: 3628-3638.

Sen S, Burmeister M, Ghosh D (2004a). Meta-analysis of the association between a serotonin transporter promoter polymorphism (5-HTTLPR) and anxiety-related personality traits. Am J Med Genet 127B: 85-89.

Sen S, Villafuerte S, Nesse R, Stoltenberg SF, Hopcian J, Gleiberman L et al (2004b). Serotonin transporter and GABAA alpha 6 receptor variants are associated with neuroticism. Biol Psychiatry 55: 244-249.

Shifman S, Bronstein M, Sternfeld M, Pisante-Shalom A, LevLehman E, Weizman A et al (2002). A highly significant association between a COMT haplotype and schizophrenia. Am J Hum Genet 71: 1296-1302.

Smith MW, Lautenberger JA, Shin HD, Chretien JP, Shrestha S, Gilbert DA et al (2001). Markers for mapping by admixture linkage disequilibrium in African American and Hispanic populations. Am J Hum Genet 69: 1080-1094.

StataCorp (2003). Stata Statistical Software (Release 8.0). Stata Corporation: College Station, TX.

Stein MB, Bienvenu OJ (2004). Diagnostic classification of anxiety disorders: DSM-V and beyond. In: Charney DS, Nestler EJ (eds). The Neurobiology of Mental Illness, 2nd edn. Oxford University Press: New York, NY. pp 525-534.

Stein MB, Chavira DA, Jang KL (2001). Bringing up bashful baby: developmental pathways to social phobia. Psychiatr Clin $\mathrm{N} \mathrm{Am}$ 24: 661-675.

Stein MB, Schork NJ, Gelernter J (2004). A polymorphism of the $\mathrm{b}_{1}$-adrenergic receptor is associated with low extraversion. Biol Psychiatry 56: 217-224.

Sullivan PF, Eaves LJ, Kendler KS, Neale MC (2001). Genetic case-control association studies in neuropsychiatry. Arch Gen Psychiatry 58: 1015-1024.

Tang H, Quertermous T, Rodriguez B, Kardia SL, Zhu X, Brown A et al (2005). Genetic structure, self-identified race/ethnicity, and confounding in case-control association studies. Am J Hum Genet 76: 268-275.

Van Gestel S, Van Broeckhoven C (2003). Genetics of personality: are we making progress? Mol Psychiatry 8: 840-852.

Winterer G, Goldman D (2003). Genetics of human prefrontal function. Brain Res Brain Res Rev 43: 134-163.

Woo JM, Yoon KS, Choi YH, Oh KS, Lee YS, Yu BH (2004). The association between panic disorder and the L/L genotype of catechol-O-methyltransferase. J Psychiatr Res 38: 365-370.

Woo JM, Yoon KS, Yu BH (2002). Catechol $O$-methyltransferase genetic polymorphism in panic disorder. Am J Psychiatry 159: 1785-1787.

Yang BZ, Zhao H, Kranzler HR, Gelernter J (2005). Practical population group assignment with selected informative markers Characteristics and properties of Bayesian clustering via STRUCTURE. Genet Epidemiol 28: 302-312. 